\title{
Visual Servoing from Spheres with Paracatadioptric Cameras
}

\author{
Romeo Tatsambon Fomena ${ }^{1}$ and François Chaumette ${ }^{2}$ \\ IRISA - Université de Rennes $1^{1}$ and INRIA ${ }^{2}$ \\ Campus de Beaulieu, 35042 Rennes cedex, France \\ firstname.name@irisa.fr
}

Summary. A paracatadioptric camera consists of the coupling of a parabolic mirror with a telecentric lens which realizes an orthographic projection to the image sensor. This type of camera provides large field of view images and has therefore potential applications for mobile and aerial robots.

This paper is concerned with visual servoing using paracatadioptric cameras. A new optimal combination of visual features is proposed for visual servoing from spheres. Using this combination, a classical control law is proved to be globally stable even in the presence of modeling error. Experimental and simulation results validate the proposed theoretical results.

\section{Introduction}

In visual servoing, data provided by a vision sensor is used to control the motion of a dynamic system [1]. A vision sensor provides a large spectrum of potential visual features. However, the use of some visual features may lead to stability problems if the displacement that the robot has to achieve is very large [2]. Therefore, there is a need to design optimal visual features for visual servoing. By optimality the satisfaction of the following criteria is meant: local and -as far as possible- global stability of the system, robustness to calibration and to modeling errors, non-singularity, local mimima avoidance, satisfactory trajectory of the system and of the features in the image, and finally a linear link and maximal decoupling between the visual features and the degrees of freedom (DOFs) taken into account.

Several approaches have been proposed to try to reach an optimal system behaviour using only $2 \mathrm{D}$ data (due to lack of space, we do not recall here the properties of pose-based visual servoing [3] and $21 / 2 \mathrm{D}$ visual servoing [4]). A satisfactory motion of the system in the cartesian space can be obtained by decoupling the $z$-axis translational and rotational motions from the other DOFs through a partitioned approach [5]. Another way around the decoupling of the optical axis motions is to use cylindrical coordinates [6]. The partitioned 
approach has been coupled with a potential function in a control scheme to keep the features in the image boundary. Potential functions can also be used in path planning in the image space to keep the features in the field of view [7]. Similarly, navigation functions can be combined with a global diffeomorphism from a visible set of rigid-body configurations of a special target to an image space, to construct global, dynamical visual servoing systems that guarantees the visibility of the features all times [8].

Central catadioptric systems (except perspective cameras), despite their more complex projection equations, are well suited for large field of view images. Considering feature points on such cameras, the interaction with the system (the link between the robot velocities and the image observations) has been shown to present the same singularities as classical perspective cameras [9]. Lately, a spherical projection model has been used to design a new minimal set of optimal visual features for visual servoing from spheres with any central catadioptric system [10]. These features mostly draw a straight line trajectory from the initial position to the desired position in the image space.

For paracatadioptric cameras, straight line trajectories are not always suitable in the image space because of the dead angle in the center of the image inherent to the physiscal realization of such systems. For this reason, there is a need to search for other features more suitable for such imaging systems. This paper presents a new optimal set of visual features for visual servoing from spheres specific to this type of cameras. This new set is built from the previous combination [10] using a cylindrical coordinate system which is appropriate to the motion of the measures in the image.

In the next section, we recall the general results concerning visual servoing from spheres using any central catadioptric system. The optimal visual features obtained from this generalization are then derived in the case of paracatadioptric cameras. In section III we propose a new optimal set of three features which is shown to be more appropriate to the feature motion in the image plane of such systems. For the proposed visual features, a theoretical analysis of the stability and the robustness of a classical control law with respect to modeling errors is given. In section IV, we validate experimentally on a paracatadioptric system the combination proposed for any central catadioptric system. Finally, simulation results are given in this same section to validate the new optimal combination.

\section{General visual features}

In this section, we recall the optimal visual features obtained for visual servoing from spheres using any central catadioptric system. These features are designed using a spherical projection model. Indeed, with this projection model, it is quite easy and intuitive to determine optimal features compared to omnidirectional projection models. 


\subsection{Spherical projection of a sphere and potential visual features}

Let $S(O, R)$ be a sphere of radius $R$ and center $O$ with coordinates $\left(X_{O}, Y_{O}, Z_{O}\right)$ in the camera frame. Let $S_{p}(C, 1)$ be the unit sphere located at the camera optical center $C$. The spherical projection of $S(O, R)$ onto $S_{p}(C, 1)$ is a dome hat [8]. This dome hat can be characterized by the contour $\Gamma$ of its base. This contour is pictured in Fig. 1(a). The analytical form of $\Gamma$ is given by

$$
\Gamma=S_{p}(C, 1) \cap \mathcal{P}=\left\{\begin{array}{l}
X_{S}^{2}+Y_{S}^{2}+Z_{S}^{2}=1 \\
X_{O} X_{S}+Y_{O} Y_{S}+Z_{O} Z_{S}=K_{O}
\end{array}\right.
$$

where $K_{O}=\sqrt{X_{O}^{2}+Y_{O}^{2}+Z_{O}^{2}-R^{2}}$. The contour $\Gamma$ is therefore a circle. Let $A$ and $r_{s}$ be respectively the center and the radius of $\Gamma$ (see Fig. 1(b)). After some developments we obtain in the camera frame

$$
\begin{gathered}
r_{s}=R / d_{O} \\
\left\{\begin{array}{l}
X_{A}=X_{O} \sqrt{1-r_{s}^{2}} / d_{O} \\
Y_{A}=Y_{O} \sqrt{1-r_{s}^{2}} / d_{O} \\
Z_{A}=Z_{O} \sqrt{1-r_{s}^{2}} / d_{O}
\end{array}\right.
\end{gathered}
$$

where $d_{O}=\sqrt{X_{O}^{2}+Y_{O}^{2}+Z_{O}^{2}}$.

In addition to $A$ and $r_{s}$, the dome hat summit $B$ (see Fig. 1(b)) can also be considered as a potential visual feature. The coordinates of $B$ in the camera frame are given by

$$
\left\{\begin{array}{l}
X_{B}=X_{O} / d_{O} \\
Y_{B}=Y_{O} / d_{O} \\
Z_{B}=Z_{O} / d_{O}
\end{array}\right.
$$

\subsection{Visual features selection}

In this section we present the interaction matrix related to the optimal visual features selected. We recall that the interaction matrix $\mathbf{L}_{\mathbf{f}}$ related to a set of features $\mathbf{f} \in \mathbb{R}^{n}$ is defined such that $\dot{\mathbf{f}}=\mathbf{L}_{\mathbf{f}} \mathbf{v}$ where $\mathbf{v}=(\boldsymbol{v}, \boldsymbol{\omega}) \in \mathfrak{s e}(\mathbf{3})$ is the instantaneous camera velocity [11]; $\boldsymbol{v}$ and $\boldsymbol{\omega}$ are respectively the translational and the rotational velocities of the camera and $\mathfrak{s e}(\mathbf{3}) \simeq \mathbb{R}^{3} \times \mathbb{R}^{3}$ is the Lie algebra of the Lie group of displacements $\mathbf{S E}(\mathbf{3})$.

Three parameters are sufficient to characterize the spherical projection of a sphere. Therefore, we need to select a combination of three visual features among $\left\{X_{A}, Y_{A}, Z_{A}, X_{B}, Y_{B}, Z_{B}, r_{s}\right\}$.

The combination $\mathbf{s}=\left(\frac{X_{B}}{r_{s}}, \frac{Y_{B}}{r_{s}}, \frac{Z_{B}}{r_{s}}\right)$ compared to the other is seductive since its interaction matrix $\mathbf{L}_{\mathbf{s}}$ is simple and maximally decoupled [10]:

$$
\mathbf{L}_{\mathbf{s}}=\left[-\frac{1}{R} \mathbf{I}_{3}[\mathbf{s}]_{\times}\right]
$$




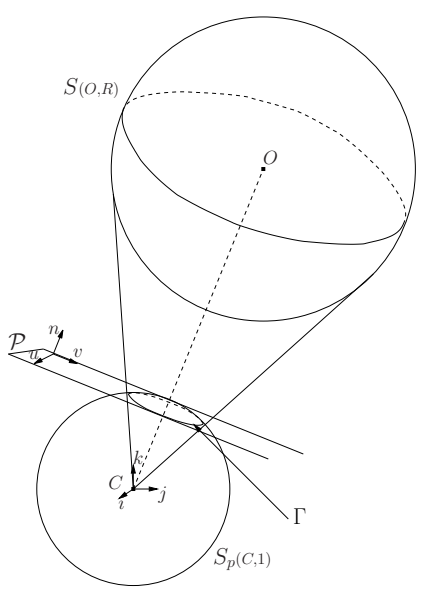

(a)

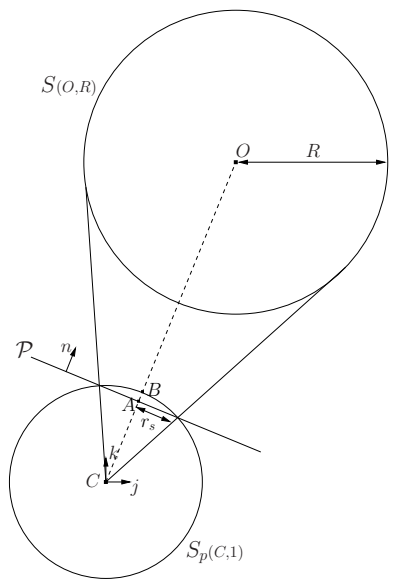

(b)

Fig. 1. Spherical projection of a sphere: (a) contour of the dome hat base; (b) cut made perpendicular to $\mathcal{P}$

In addition to the decoupling property, $\mathbf{L}_{\mathbf{s}}$ presents the same dynamic $\left(\frac{1}{R}\right)$ in the translational velocities. Since $R$ is a constant, there is a linear link between the visual features and the camera translational velocities. We can also see that the interaction matrix presents the passivity property, which is important to control certain under-actuated systems [12]. For these reasons, we propose the combination $\mathbf{s}=\left(\frac{X_{B}}{r_{s}}, \frac{Y_{B}}{r_{s}}, \frac{Z_{B}}{r_{s}}\right)$ for visual servoing from spheres.

The only unknown 3D parameter in $\mathbf{L}_{\mathrm{s}}$ is the constant $R$. In practice, $\widehat{R}$ (estimated value of $R$ ) is used instead. The robustness domain of a classical control law has been shown in [10] to be extremely large: $\widehat{R} \in] 0,+\infty[$. Therefore, from a practical point of view, a rough estimate of $R$ is sufficient.

We will now show how to compute this set of features using any central catadioptric system.

\subsection{Visual features computation using any central catadioptric system}

Considering a catadioptric system with $(\varphi, \xi)$ as the mirror parameters, we show in this section that we can compute the visual features $\mathbf{s}=\left(\frac{X_{B}}{r_{s}}, \frac{Y_{B}}{r_{s}}, \frac{Z_{B}}{r_{s}}\right)$ from the catadioptric image of a sphere.

The catadioptric image of a sphere is an ellipse. Ellipse formation can be decomposed in two steps (see Fig. 2(a)) considering the unified model of catadioptric image formation [13]. From Fig. 2, note that the unique viewpoint is $V$ and the camera optical center is $C$.

The first step is the spherical projection of $S(O, R)$ onto $S_{p}(V, 1)$. This result has been presented in section 2.1. Since $S(O, R)$ is described in the virtual 


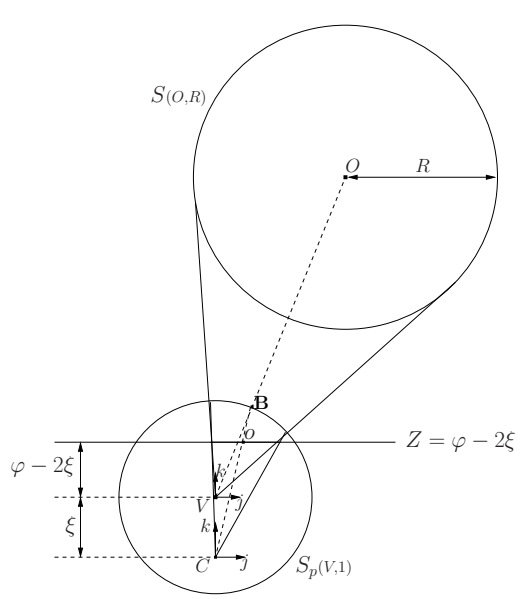

(a)

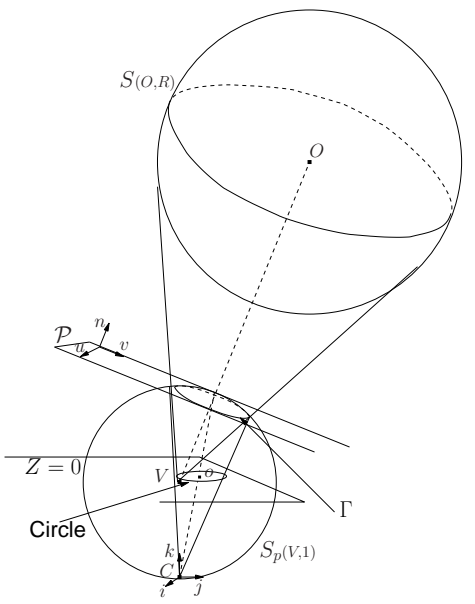

(b)

Fig. 2. Central catadioptric image of a sphere: (a) general case; (b) paracatadioptric projection $(\xi=1)$

frame centered in $V$, we obtain

$$
\Gamma=\left\{\begin{array}{l}
X_{S}^{V^{2}}+Y_{S}^{V^{2}}+Z_{S}^{V^{2}}=1 \\
X_{O} X_{S}^{V}+Y_{O} Y_{S}^{V}+Z_{O} Z_{S}^{V}=K_{O} .
\end{array}\right.
$$

$\Gamma$ is then expressed in the camera frame and projected onto the catadioptric image plane $Z=\varphi-2 \xi$. $\Gamma$ is therefore the intersection of the sphere

$$
X_{S}^{2}+Y_{S}^{2}+\left(Z_{S}-\xi\right)^{2}=1
$$

with the plane

$$
X_{O} X_{S}+Y_{O} Y_{S}+Z_{O} Z_{S}=K_{O}+\xi Z_{O}
$$

The equations of projection onto the catadioptric image plane are nothing but

$$
\left\{\begin{array}{l}
x=\frac{X_{S}}{Z_{S}} \\
y=\frac{Y_{S}}{Z_{S}}
\end{array}\right.
$$

Plugging (9) in (8) gives

$$
\frac{1}{Z_{S}}=\frac{X_{O} x+Y_{O} y+Z_{O}}{K_{O}+\xi Z_{O}}
$$

and (9) in (7) gives

$$
x^{2}+y^{2}+1-2 \frac{\xi}{Z_{S}}+\frac{\xi^{2}-1}{Z_{S}^{2}}=0 .
$$


Finally, injecting (10) in (11) leads to the ellipse equation

$$
\begin{gathered}
k_{0} x^{2}+k_{1} y^{2}+2 k_{2} x y+2 k_{3} x+2 k_{4} y+k_{5}=0 \\
\text { with }\left\{\begin{array}{l}
k_{0}=\left(K_{O}+\xi Z_{O}\right)^{2}+\left(\xi^{2}-1\right) X_{O}^{2} \\
k_{1}=\left(K_{O}+\xi Z_{O}\right)^{2}+\left(\xi^{2}-1\right) Y_{O}^{2} \\
k_{2}=\left(\xi^{2}-1\right) X_{O} Y_{O} \\
k_{3}=X_{O}\left(\left(\xi^{2}-1\right) Z_{O}-\xi\left(K_{O}+\xi Z_{O}\right)\right) \\
k_{4}=Y_{O}\left(\left(\xi^{2}-1\right) Z_{O}-\xi\left(K_{O}+\xi Z_{O}\right)\right) \\
k_{5}=\left(K_{O}+\xi Z_{O}\right)^{2}+\left(\xi^{2}-1\right) Z_{O}^{2}-2 \xi Z_{O}\left(K_{O}+\xi Z_{O}\right) .
\end{array}\right.
\end{gathered}
$$

Now, we show how to compute $\mathbf{s}$ using the ellipse moments $\mu=\left(x_{g}, y_{g}, n_{20}, n_{11}, n_{02}\right)$ measured on the catadioptric image plane: $\left(x_{g}, y_{g}\right)$ is the ellipse center of gravity; $n_{02}$ and $n_{20}$ are the ellipse axes length and $n_{11}$ is equivalent to the ellipse orientation.

First of all, we recall that:

$$
\left\{\begin{array}{l}
\frac{X_{B}}{r_{s}}=\frac{X_{O}}{R} \\
\frac{Y_{B}}{r_{s}}=\frac{Y_{O}}{R} \\
\frac{Z_{B}}{r_{s}}=\frac{Z_{O}}{R}
\end{array}\right.
$$

From (12), the ellipse moments on the catadioptric image plane can be expressed using the $3 \mathrm{D}$ parameters:

$$
\begin{gathered}
\left\{\begin{array}{l}
x_{g}=X_{O} H_{1} / H_{2} \\
y_{g}=Y_{O} H_{1} / H_{2} \\
4 n_{20}=\left(H_{2}-\left(\xi^{2}-1\right) X_{O}^{2}\right) R^{2} / H_{2}^{2} \\
4 n_{11}=-X_{O} Y_{O}\left(\xi^{2}-1\right) R^{2} / H_{2}^{2} \\
4 n_{02}=\left(H_{2}-\left(\xi^{2}-1\right) Y_{O}^{2}\right) R^{2} / H_{2}^{2}
\end{array}\right. \\
\text { with }\left\{\begin{array}{l}
H_{1}=Z_{O}+\xi K_{O} \\
H_{2}=H_{1}^{2}+\left(\xi^{2}-1\right) R^{2} .
\end{array}\right.
\end{gathered}
$$

After tedious computations, we obtain using (14)

$$
\left\{\begin{array}{l}
\frac{X_{B}}{r_{s}}=x_{g} \frac{h_{2}}{\sqrt{h_{2}+\left(1-\xi^{2}\right)}} \\
\frac{Y_{B}}{r_{s}}=y_{g} \frac{h_{2}}{\sqrt{h_{2}+\left(1-\xi^{2}\right)}}
\end{array}\right.
$$

where $h_{2}=1 / f(\mu)$ with $f(\mu)=\frac{4 n_{20} y_{g}^{2}+4 n_{02} x_{g}^{2}-8 n_{11} x_{g} y_{g}}{x_{g}^{2}+y_{g}^{2}}$.

It is possible to demonstrate that $f(\mu)$ is continuous even when $x_{g}=y_{g}=0$ in which case $f(\mu)=4 n_{20}$.

In the case of paracatadioptric systems (see Fig. 2(b)) where $h_{2}=1 / \sqrt{4 n_{20}}$, we also obtain:

$$
\frac{Z_{B}}{r_{s}}=\frac{h_{2}-\left(\frac{X_{B}^{2}}{r_{s}^{2}}+\frac{Y_{B}^{2}}{r_{s}^{2}}-1\right)}{2 \sqrt{h_{2}}},
$$


and for all other catadioptric systems $(\xi \neq 1)$

$$
\frac{Z_{B}}{r_{s}}=\frac{h_{1}-\xi \sqrt{h_{1}^{2}+\left(1-\xi^{2}\right)\left(\frac{X_{B}^{2}}{r_{s}^{2}}+\frac{Y_{B}^{2}}{r_{s}^{2}}-1\right)}}{\left(1-\xi^{2}\right)}
$$

where $h_{1}=\sqrt{h_{2}+\left(1-\xi^{2}\right)}$.

The features $\mathbf{s}=\left(\frac{X_{B}}{r_{s}}, \frac{Y_{B}}{r_{s}}, \frac{Z_{B}}{r_{s}}\right)$ are intuitively proper to a cartesian image space. Therefore, for any visual servoing task, these features will mostly draw a straight line trajectory in the image plane of any catadioptric system. This is not always suitable for paracatadioptric cameras since there is a dead angle in the centre of the image. Therefore we present, in the next section, a new optimal combination for such cameras.

\section{Optimal visual features}

The new combination proposed here is shown to be more suitable with the physical realization of such cameras. In addition, the stability of the system is analysed: a sufficient condition is given for the global stability of the system with respect to modeling error.

\subsection{Optimal features design}

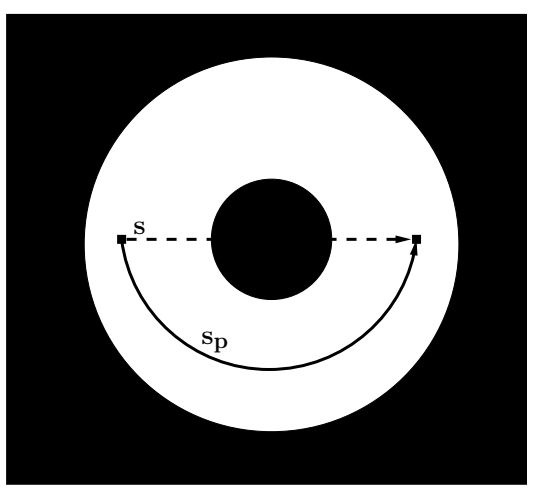

Fig. 3. Coordinate system dependence of the features motion

Let us consider a task of visual servoing from a sphere using a paracatadioptric camera where the initial and desired positions (of the center of gravity of the sphere image) are the mirror image of each other. Using the general features $\mathbf{s}=\left(\frac{X_{B}}{r s}, \frac{Y_{B}}{r_{s}}, \frac{Z_{B}}{r_{s}}\right)$ will lead to a straight line features motion and thus to the loss of the target in the dead angle (in the center of the image) as 
shown in Fig. 3. Since this dead angle is inherent to the physical realization of a paracatadioptric camera, we propose to use the cylindrical coordinates of $\left(\frac{X_{B}}{r s}, \frac{Y_{B}}{r_{s}}\right)$; this will prevent the loss of the target in the dead angle by enforcing a circular feature motion (see Fig. 3). Therefore, the new optimal visual features $\mathbf{S}_{\mathbf{p}}$ computed from $\mathbf{s}$ are given by

$$
\left\{\begin{array}{l}
\rho=\sqrt{\left(\frac{X_{B}}{r_{s}}\right)^{2}+\left(\frac{Y_{B}}{r_{s}}\right)^{2}} \\
\theta=\arctan \frac{Y_{B}}{X_{B}} \\
\frac{Z_{B}}{r_{s}}=\frac{h_{2}-\left(\frac{X_{B}^{2}}{r_{s}^{2}}+\frac{Y_{B}^{2}}{r_{s}^{2}}-1\right)}{2 \sqrt{h_{2}}}
\end{array}\right.
$$

where $h_{2}=\frac{1}{4 n_{20}}$.

In addition to the better feature motion in the image, it is important to note that the feature $\rho$ can never be 0 on a paracatadioptric image plane since the region where the target is visible does not include the center of the image. Thus $\theta$ is always defined.

The interaction matrix related to $\mathbf{s}_{\mathbf{p}}$ is given by

$$
\mathbf{L}_{\mathbf{s}_{\mathbf{p}}}=\left[\begin{array}{cccccc}
\frac{-c}{R} & \frac{-s}{R} & 0 & \frac{s Z_{B}}{r_{s}} & \frac{-c Z_{B}}{r_{s}} & 0 \\
\frac{s}{\rho R} & \frac{-c}{\rho R} & 0 & \frac{c Z_{B}}{r_{s} \rho} & \frac{s Z_{B}}{r_{s} \rho} & -1 \\
0 & 0 & -\frac{1}{R} & -\rho s & \rho c & 0
\end{array}\right],
$$

with $c=\cos \theta$ et $s=\sin \theta$.

From this interaction matrix, we can see that $\frac{Z_{B}}{r_{r}}$ is the only feature that is sensitive to the $z$-translation while $\theta$ is the only feature related to the rotation around the optical axis. This constrains the feature motion to avoid the dead angle. For these reasons, we propose the combination $\left(\rho, \theta, \frac{Z_{B}}{r_{s}}\right)$ for visual servoing from spheres using paracatadioptric cameras.

The only unknown 3D parameter in $\mathbf{L}_{\mathbf{s}_{\mathbf{p}}}$ is still the constant $R$. As before, in practice, $\widehat{R}$ ( the estimated value of $R$ ) is used instead. From the stability analysis to modeling error, a robustness domain of $\widehat{R}$ will be given.

\subsection{Stability analysis to modeling error}

Let us consider visual servoing from spheres with the combination $\mathbf{s}_{\mathbf{p}}=$ $\left(\rho, \theta, \frac{Z_{B}}{r_{s}}\right)$.

We use the classical control law

$$
\mathbf{v}_{c}=-\lambda{\widehat{\mathbf{L}_{\mathbf{p}}}}^{+}\left(\mathbf{s}_{\mathbf{p}}-\mathbf{s}_{\mathbf{p}}{ }^{*}\right)
$$

where $\mathbf{v}_{c}$ is the camera velocity sent to the low level robot controller, $\lambda$ is a positive gain and ${\widehat{\mathbf{L}_{\mathbf{s}_{\mathrm{p}}}}}^{+}$is the pseudo-inverse of an approximation of the interaction matrix related to $\mathbf{s}_{\mathbf{p}}$. 
Modeling error arises from the approximation of $R$. In this case the closedloop system equation can be written as:

$$
\dot{\mathbf{s}}_{\mathbf{p}}=-\lambda \mathbf{L}_{\mathbf{s}_{\mathbf{p}}}{\widehat{\mathbf{L}_{\mathbf{p}}}}^{+}\left(\mathbf{s}_{\mathbf{p}}-\mathbf{s}_{\mathbf{p}}{ }^{*}\right)
$$

where

$$
\widehat{\mathbf{L}}_{\mathbf{S}_{\mathbf{p}}}^{+}=\left[\begin{array}{ccc}
\frac{-c \hat{R}\left(\rho^{2} \hat{R}^{2}+1\right)}{d} & \frac{\rho s \hat{R}}{d} & \frac{-\rho c Z_{B} \hat{R}^{3}}{d r_{s}} \\
\frac{-s \hat{R}\left(\rho^{2} \hat{R}^{2}+1\right)}{d} & \frac{-\rho c \hat{R}}{d} & \frac{-\rho s Z_{B} \hat{R}^{3}}{d r_{s}} \\
\frac{-\rho Z_{B} \hat{R}^{3}}{d r_{s}} & 0 & \frac{-\hat{R}\left(1+\left(\frac{Z_{B}}{r_{s}}\right)^{2} \hat{R}^{2}\right)}{d} \\
\frac{s Z_{B} \hat{R}^{2}}{d r_{s}} & \frac{\rho c Z_{B} \hat{R}^{2}}{d r_{s}} & \frac{-\rho s \hat{R}^{2}}{d} \\
\frac{-c Z_{B} \hat{R}^{2}}{d r_{s}} & \frac{\rho s Z_{B} \hat{R}^{2}}{d r_{s}} & \frac{\rho c \hat{R}^{2}}{d} \\
0 & -\frac{\rho^{2} \hat{R}^{2}}{d} & 0
\end{array}\right]
$$

with $d=1+\left(\left(\frac{Z_{B}}{r_{s}}\right)^{2}+\rho^{2}\right) \hat{R}^{2}$. A sufficient condition for the global asymptotic stability to modeling error is $\mathbf{L}_{\mathbf{s}_{\mathbf{p}}}{\widehat{\mathbf{L}_{\mathbf{p}}}}^{+}>0$. The eigenvalues of $\mathbf{L}_{\mathbf{s}_{\mathrm{p}}}{\widehat{\mathbf{L}_{\mathbf{s}_{\mathrm{p}}}}}^{+}$ can be computed. They are given by $\frac{\hat{R}}{R}$ and $\frac{\hat{R}}{R} \frac{r_{s}^{2}+R \hat{R} Z_{B}^{2}+R \hat{R} r_{s}^{2} \rho^{2}}{r_{s}^{2}+\hat{R}^{2} Z_{B}^{2}+\hat{R}^{2} r_{s}^{2} \rho^{2}}$ (which is a double eigenvalue). We have thus:

$$
\mathbf{L}_{\mathbf{s}_{\mathbf{p}}}{\widehat{\mathbf{L}_{\mathbf{s}_{\mathbf{p}}}}}^{+}>0 \Longleftrightarrow \widehat{R}>0
$$

This condition is also necessary since if $\widehat{R} \leq 0$ then $\mathbf{L}_{\mathbf{s}} \widehat{\mathbf{L}}_{\mathbf{s}}^{+} \leq 0$ and the system diverges. Therefore the robustness domain with respect to modeling error is: $\widehat{R} \in] 0,+\infty\left[\right.$. This result is not a surprise at all since $\mathbf{s}_{\mathbf{p}}$ has been computed from $\mathbf{s}$ through a bijective map. From a practical point of view, a coarse approximation of $R$ will thus be sufficient.

\section{Results}

In this section we first validate the general features $\mathbf{s}=\left(\frac{X_{B}}{r_{s}}, \frac{Y_{B}}{r_{s}}, \frac{Z_{B}}{r_{s}}\right)$ on a real robotic system using a paracatadioptric camera. Then we show in simulation that these features, for a particular simple visual servoing task, draw a highly undesirable straight line trajectory in the image plane. We finally validate the new optimal features $\mathbf{s}_{\mathbf{p}}=\left(\rho, \theta, \frac{Z_{B}}{r_{s}}\right)$ in simulation.

\subsection{Experimental results}

In this section, the general features $\mathbf{s}$ are validated. The experiments have been carried out with a paracatadioptric camera mounted on the end-effector of a six DOFs robotic system. The target is a $4 \mathrm{~cm}$ radius polystyrene white 
ball. Using such a simple object allows to easily compute the ellipse moments at video rate without any image processing problem. The desired set $\mathbf{s}^{*}$ has been computed after moving the robot to a position corresponding to the desired image. Fig. 4 shows the desired and the initial images used for each experiment. For all the experiments, the same gain $\lambda=0.1$ has been used.

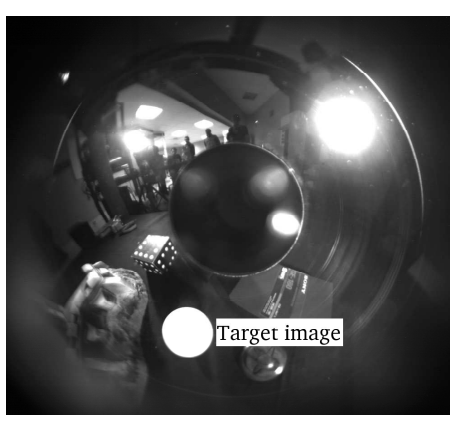

(a)

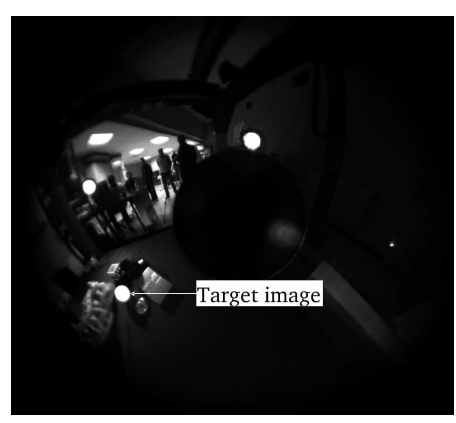

(b)

Fig. 4. (a) Desired image; (b) initial image

\section{Ideal case}

In oder to validate the general features $\mathbf{s}$, we first consider the ideal case where $\widehat{R}=R$. Indeed, when $\widehat{R}=R$ we have a perfect system behaviour since $\mathbf{L}_{\mathbf{s}} \widehat{\mathbf{L}}_{\mathbf{s}}^{+}=\mathbf{I}_{3}$. As expected, a pure exponential decrease of the error on the visual features can be observed on Fig. 5(a) while the camera velocities are plotted on Fig. 5(b).

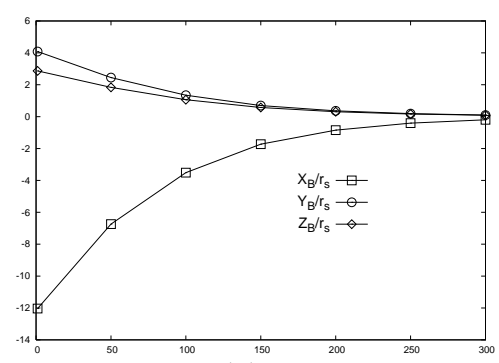

(a)

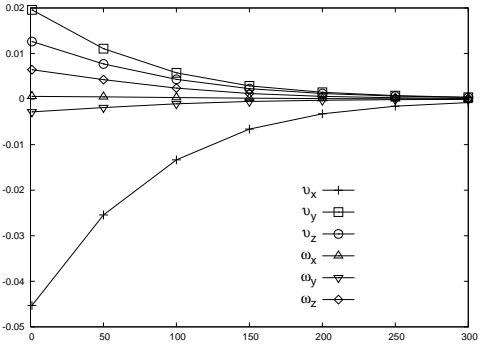

(b)

Fig. 5. Ideal case: (a) s error; (b) computed camera velocities $(\mathrm{m} / \mathrm{s}$ and $\mathrm{dg} / \mathrm{s})$ 


\section{Modeling error}

The stability with respect to modeling error using $\mathbf{s}$ has been proved using a classical perspective camera [10]. For paracatadioptric system, we have validated this proof, with two experiments. The results in the case where $\widehat{R}=5 R$ and $\widehat{R}=0.2 R$ are depicted respectively in Fig. 6 and Fig. 7 . We can note that the system still converges in both cases.

Fig. 6(b) shows a high speed on the system translational velocities while Fig. 7(b) shows a low speed on the same components. In fact, choosing an arbitrary value of $\widehat{R}$ affects the convergence speed of the system. Indeed, using the general features $\mathbf{s}$, the velocity sent to the robot can be written as

$$
\mathbf{v}_{c}=-\lambda \widehat{\mathbf{L}}_{\mathbf{s}}^{+}\left(\mathbf{s}-\mathbf{s}^{*}\right)
$$

where $\widehat{\mathbf{L}}_{\mathbf{s}}^{+}$computed from (5) is given by

$$
\widehat{\mathbf{L}}_{\mathbf{s}}^{+}=\left[\begin{array}{c}
-\frac{\widehat{R} r_{s}^{2}}{r_{s}^{2}+\widehat{R}^{2}}\left(\widehat{R}^{2} \mathbf{s} \mathbf{s}^{\top}+\mathbf{I}_{3}\right) \\
-\frac{\widehat{R}^{2} r_{s}^{2}}{r_{s}^{2}+\widehat{R}^{2}}[\mathbf{s}]_{\times}
\end{array}\right] .
$$

After few developments we obtain from (21)

$$
\left\{\begin{array}{l}
\boldsymbol{v}=\lambda \frac{\widehat{R} r_{s}^{2}}{r_{s}^{2}+\widehat{R}^{2}}\left(\widehat{R}^{2} \mathbf{s s}^{\top}+\mathbf{I}_{3}\right)\left(\mathbf{s}-\mathbf{s}^{*}\right) \\
\boldsymbol{\omega}=\lambda \frac{\widehat{R}^{2} r_{s}^{2}}{r_{s}^{2}+\widehat{R}^{2}}[\mathbf{s}]_{\times}\left(\mathbf{s}-\mathbf{s}^{*}\right) .
\end{array}\right.
$$

When $\widehat{R}$ tends to $+\infty$, (22) tends to

$$
\left\{\begin{array}{l}
\boldsymbol{v}=\infty \\
\boldsymbol{\omega}=\lambda r_{s}^{2}[\mathbf{s}]_{\times}\left(\mathbf{s}-\mathbf{s}^{*}\right)
\end{array}\right.
$$

which explains the fast convergence observed in Fig. 6 (100 iterations) when $\widehat{R}=5 R$. When $\widehat{R}$ tends to 0 , from (22) we have: $\boldsymbol{v}$ and $\boldsymbol{\omega}$ tend to 0 . This explains the slow convergence observed in Fig. 7 when $\widehat{R}=0.2 R$. In practice, the behaviour could be easily improved, by using a higher gain $\lambda$ (to deal with under approximation of $\widehat{R}$ ) and by saturating $\mathbf{v}_{c}$ when needed (to deal with an over approximation of $\widehat{R}$ ).

\subsection{Simulation results}

In this section, it is shown that for the general feature $\mathbf{s}$, the motion in the image plane is not suitable with paracatadioptric cameras, particularly when the initial position and desired position (in the image space) are each others mirror image. In addition, the new optimal features $\mathbf{s}_{\mathbf{p}}$ specific to the paracatadioptric system are validated. 


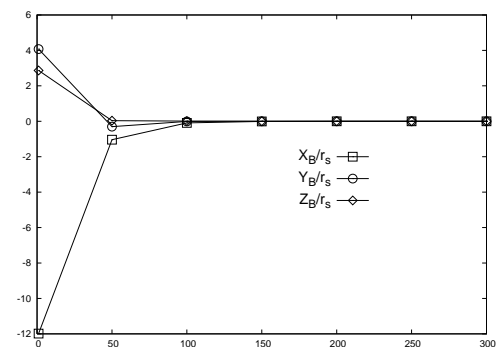

(a)

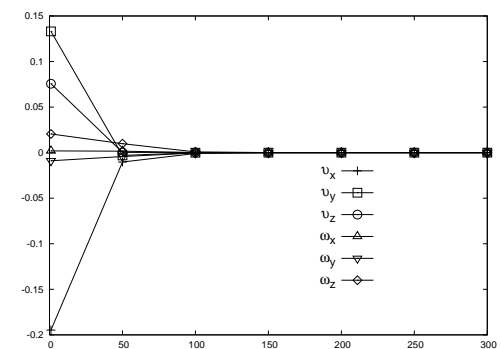

(b)

Fig. 6. Modeling error $\widehat{R}=5 R$ : (a) s error; (b) computed camera velocities $(\mathrm{m} / \mathrm{s}$ and $\mathrm{dg} / \mathrm{s})$

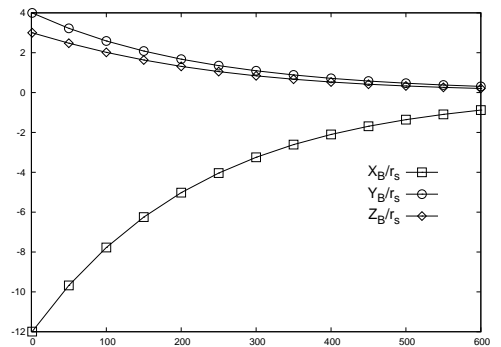

(a)

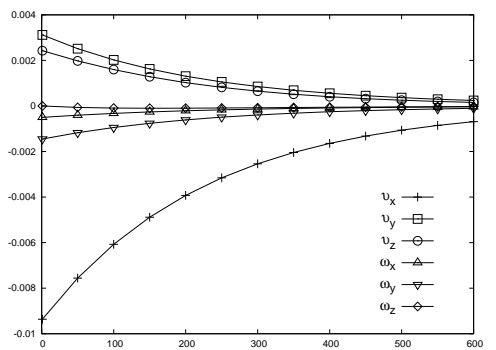

(b)

Fig. 7. Modeling error $\widehat{R}=0.2 R$ : (a) s error; (b) computed camera velocities $(\mathrm{m} / \mathrm{s}$ and $\mathrm{dg} / \mathrm{s}$ )

\section{Features motion in the image plane}

Here we consider a visual servoing task where the initial and desired images are each the mirror image of the other (rotation of $\pi$ around the $z$-axis). The image-plane trajectories of the center of gravity of the sphere image are drawn in Fig. 8(c). In this picture we can see that the general features $\mathbf{s}$ generate a straight line motion going through the center of the image. It means that in case of a real camera, the target would get lost in the dead angle.

Using the new features $\mathbf{s}_{\mathbf{p}}$ leads to a circular trajectory as expected. It means that with a real camera, it is possible to constrain $\rho$ to avoid the dead angle.

For all the following experiments, we consider a more complex task consisting of the previous task, a zoom and a translation in the $\rho$-direction. 


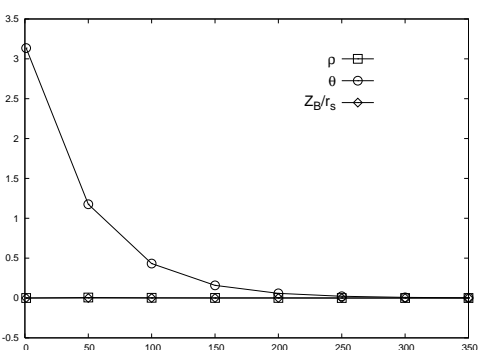

(a)



(b)

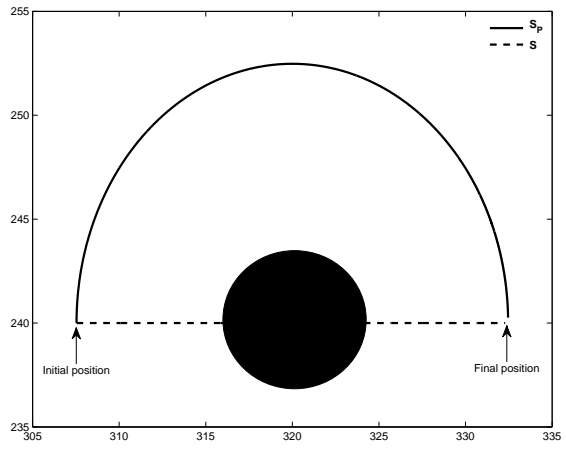

(c)

Fig. 8. Adequate features for paracatadioptric cameras: (a) $\mathbf{s}_{\mathbf{p}}$ error; (b) s error; (c) image-plane trajectories of the center of gravity of the sphere image

\section{Ideal case}

We first consider the case where $\widehat{R}=R$. In this case we have $\mathbf{L}_{\mathbf{s}_{\mathbf{p}}}{\widehat{\mathbf{L}} \mathbf{s}_{\mathbf{p}}}^{+}=\mathbf{I}_{3}$, thus a perfect system behaviour. Fig. 9 (a) plots the features error trajectory while Fig. 9(b) shows the camera velocities.

\section{Modeling error}

The stability to modeling error has been proved in this paper. This proof is validated with two experiments. In the first case, $\widehat{R}=5 R$ : Fig. 10 plots the results. In the second case, $\widehat{R}=0.2 R$ : Fig. 11 shows the results. In both cases the system still converges either fastly or slowly as expected.

\section{Calibration errors}

Finally we verify the stability to calibration errors in simulation. This is done by introducing errors on the camera intrinsic parameters: $35 \% f,-25 \% u_{0}$ and 


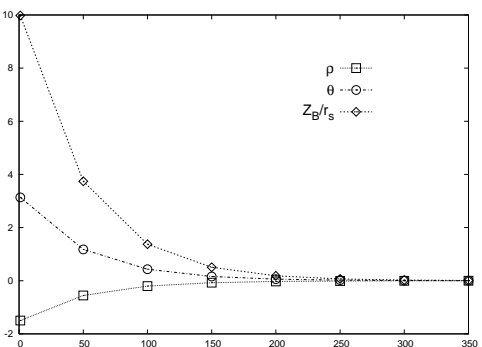

(a)

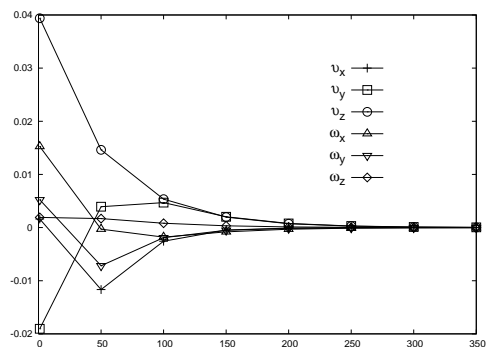

(b)

Fig. 9. Ideal case: (a) $\mathbf{s}_{\mathbf{p}}$ error; (b) computed camera velocities $(\mathrm{m} / \mathrm{s}$ and $\mathrm{dg} / \mathrm{s})$

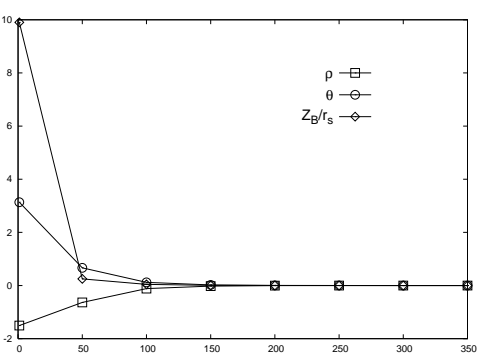

(a)

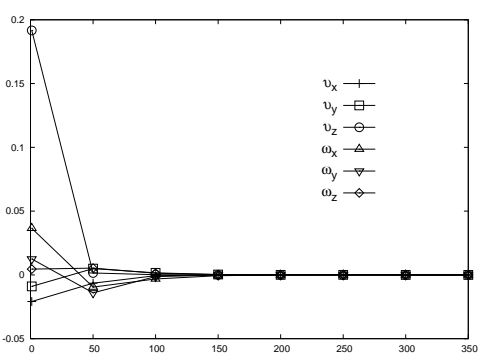

(b)

Fig. 10. Modeling error $\widehat{R}=5 R$ : (a) $\mathbf{s}_{\mathbf{p}}$ error; (b) computed camera velocities $(\mathrm{m} / \mathrm{s}$ and $\mathrm{dg} / \mathrm{s}$ )

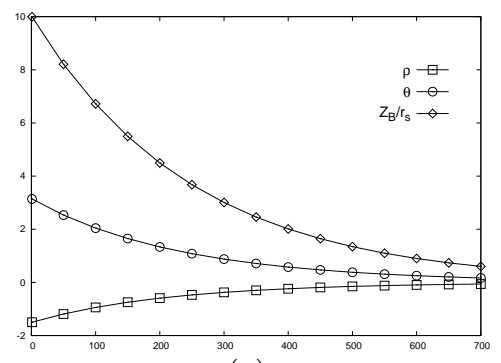

(a)

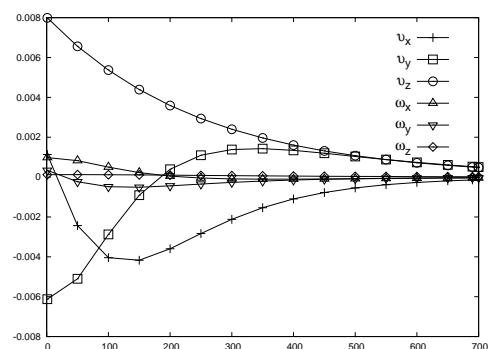

(b)

Fig. 11. Modeling error $\widehat{R}=0.2 R$ : (a) $\mathbf{s}_{\mathbf{p}}$ error; (b) computed camera velocities $(\mathrm{m} / \mathrm{s}$ and $\mathrm{dg} / \mathrm{s})$ 


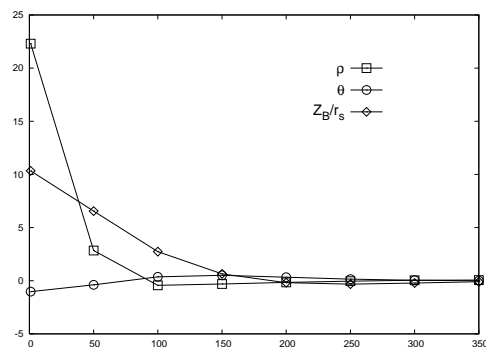

(a)

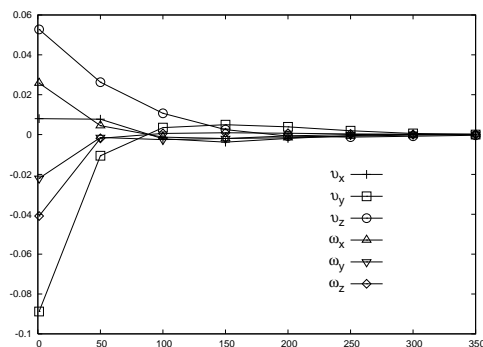

(b)

Fig. 12. Calibration errors: (a) $\mathbf{s}_{\mathbf{p}}$ error; (b) computed camera velocities $(\mathrm{m} / \mathrm{s}$ and $\mathrm{dg} / \mathrm{s})$

$47 \% v_{0}$. The results obtained are given on Fig. 12. Once again the system still converges.

\section{Conclusions}

In this paper, we have reviewed the general features designed using a spherical projection model for visual servoing from spheres with any central catadioptric system. These features usually draw a straight line trajectory in the image space which is not always suitable for paracatadioptric cameras. A new optimal combination of three visual features for visual servoing from spheres using this type of cameras has been proposed. This new set of features has been built from the previous one using a cylindrical coordinate system which enables a better feature motion in the image plane. The interaction matrix related to this new combination presents a decoupling between the rotational and the translational velocities of the optical axis. Using this new combination, a classical control law has been analytically proved to be globally stable with respect to modeling error. The general visual features have been validated experimentally with a paracatadioptric camera mounted on a robotic system and simulation results have been presented to validate the new combination.

\section{Acknowledgment}

The authors would like to thank C. Collewet, N. Mansard and S. Hutchinson for their helpful comments. 


\section{References}

1. S. Hutchinson, G. Hager, and P. Corke, "A tutorial on visual servo control," IEEE Trans. on Robotics and Automation, vol. 12, no. 3, pp. 651-670, Oct. 1996.

2. F. Chaumette, "Potential problems of stability and convergence in image-based and position-based visual servoing," in The Confluence of Vision and Control, D. Kriegman, G. Hager, and A. S. Morse, Eds. LNCIS Series, No 237, SpringerVerlag, 1998, pp. 66-78.

3. W. Wilson, C. Hulls, and G. Bell, "Relative end-effector control using cartesian position-based visual servoing," IEEE Trans. on Robotics and Automation, vol. 12 , no. 5, pp. 684-696, Oct. 1996.

4. E. Malis, F. Chaumette, and S. Boudet, "2 1/2 d visual vervoing," IEEE Trans. on Robotics and Automation, vol. 15, no. 2, pp. 238-250, Apr. 1999.

5. P. Corke and S. Hutchinson, "A new partitioned approach to image-based visual visual servo control," IEEE Trans. on Robotics and Automation, vol. 17, no. 4, pp. 507-515, Aug. 2001.

6. M. Iwatsuki and N. Okiyama, "A new formulation for visual servoing based on cylindrical coordinate system," IEEE Trans. on Robotics, vol. 21, no. 2, pp. 266-273, Apr. 2005.

7. Y. Mezouar and F. Chaumette, "Path planning for robust image-based control," IEEE Trans. on Robotics and Automation, vol. 18, no. 4, pp. 534-549, Aug. 2002.

8. N. Cowan and D. Chang, "Geometric visual servoing," IEEE Trans. on Robotics, vol. 21, no. 6, pp. 1128-1138, Dec. 2005.

9. J. Barreto, F. Martin, and R. Horaud, "Visual servoing/tracking using central catadioptric images," in Int. Symposium on Experimental Robotics, Ischia, Italy, Jul. 2002.

10. R. Tatsambon Fomena and F. Chaumette, "Visual servoing from spheres using a spherical projection model," in IEEE Int. Conf. on Robotics and Automation, Rome, Italy, Apr. 2007.

11. B. Espiau, F. Chaumette, and P. Rives, "A new approach to visual servoing in robotics," IEEE Trans. on Robotics and Automation, vol. 8, no. 3, pp. 313-326, Jun. 1992.

12. T. Hamel and R. Mahony, "Visual servoing of an under-actuated dynamic rigidbody system: an image-based approach," IEEE Trans. on Robotics and Automation, vol. 18, no. 2, pp. 187-198, Apr. 2002.

13. C. Geyer and K. Daniilidis, "A unifying theory for central panoramic systems and practical implications," European Conference on Computer Vision, vol. 29, pp. 159-179, May 2000. 